\title{
Analysis of De Novo HOXA13 Polyalanine Expansions Supports Replication Slippage Without Repair in Their Generation
}

\author{
Kailey M. Owens, ${ }^{1}$ Shane C. Quinonez, ${ }^{1}$ Peedikayil E. Thomas, ${ }^{2}$ Catherine E. Keegan, ${ }^{1,2}$ Nanci Lefebvre, ${ }^{3}$ \\ Diane Roulston, ${ }^{3}$ Christine A. Larsen, ${ }^{4}$ H. Scott Stadler, ${ }^{4}$ and Jeffrey W. Innis ${ }^{1,2 *}$ \\ ${ }^{1}$ Department of Pediatrics and Communicable Diseases, University of Michigan, Ann Arbor, Michigan \\ ${ }^{2}$ Department of Human Genetics, University of Michigan, Ann Arbor, Michigan \\ ${ }^{3}$ Department of Pathology, Cytogenetics Laboratory, University of Michigan, Ann Arbor, Michigan \\ ${ }^{4}$ Department of Molecular and Medical Genetics, Oregon Health Sciences University and Shriners Hospital for Children, Portland, Oregon
}

Manuscript Received: 3 October 2012; Manuscript Accepted: 12 December 2012

Polyalanine repeat expansion diseases are hypothesized to result from unequal chromosomal recombination, yet mechanistic studies are lacking. We identified two de novo cases of handfoot-genital syndrome (HFGS) associated with polyalanine expansions in HOXA13 that afforded rare opportunities to investigate the mechanism. The first patient with HFGS was heterozygous for a de novo nine codon polyalanine expansion. Haplotype investigation showed that the expansion arose on the maternally inherited chromosome but not through unequal crossing over between homologs, leaving unequal sister chromatid exchange during mitosis or meiosis or slipped mispairing as possible explanations. The asymptomatic father of the second patient with HFGS was mosaic for a six codon polyalanine expansion. Multiple tissue PCR and clonal analysis of paternal fibroblasts showed only expansion/WT and WT/WT clones, and haplotype data showed that two unaffected offspring inherited the same paternal allele without the expansion, supporting a postzygotic origin. Absence of the contracted allele in the mosaic father does not support sister chromatid exchange in the origin of the expansion. Mosaicism for HOXA13 polyalanine expansions may be associated with a normal phenotype, making examination of parental DNA essential in apparently de novo HFGS cases to predict accurate recurrence risks. We could not find an example in the literature where unequal sister chromatid exchange has been proven for any polyalanine expansion, suggesting that the principal mechanism for polyalanine expansions (and contractions) is slipped mispairing without repair or that the true frequency of unequal sister chromatid exchange involving these repeats is low. $\odot 2013$ Wiley Periodicals, Inc.

Key words: hand-foot-genital syndrome; HOXA13; polyalanine expansion

\section{INTRODUCTION}

Hand-foot-genital syndrome (HFGS; OMIM 140000) is an autosomal dominant condition characterized by limb and urogenital
How to Cite this Article:

Owens KM, Quinonez SC, Thomas PE, Keegan CE, Lefebvre N, Roulston D, Larsen CA, Stadler HS, Innis JW. 2013. Analysis of de novo HOXA13 polyalanine expansions supports replication slippage without repair in their generation.

Am J Med Genet Part A 161A:1019-1027.

defects, including bilateral shortening of the thumbs and great toes, vesicoureteral reflux, incomplete Müllerian fusion in females, and hypospadias in males [Innis, 2006]. The HFGS is caused by mutations in the HOXA13 gene (OMIM 142959) [Mortlock and Innis, 1997; Goodman, 2000]. The HOXA13 gene includes two exons; exon one contains three large polyalanine repeat tracts of 14,12 , and 18 residues (labeled I through III for simplicity), while exon two encodes the homeodomain. The majority of reported mutations in the HOXA13 gene in patients with HFGS have been in-frame, meiotically and mitotically stable, short (7-14 residue) expansions within any one of the three large polyalanine tracts, while point mutations comprise nearly all of the remaining mutations [Innis, 2006].

At least nine distinct diseases are associated with short polyalanine expansions in other genes encoded by imperfect triplet

Grant sponsor: University of Michigan, Department of Human Genetics and the Department of Pediatrics.

None of the authors have any conflicts of interest related to this publication. ${ }^{\star}$ Correspondence to:

Jeffrey W. Innis, 4909 Buhl Building, 1241 E. Catherine Street, Ann Arbor, MI 48109-5618. E-mail: innis@umich.edu

Article first published online in Wiley Online Library

(wileyonlinelibrary.com): 26 March 2013

DOI 10.1002/ajmg.a.35843 
repeats [Brown and Brown, 2004], and recently NIPA1 polyalanine expansions have been associated with earlier onset and shortened median survival in amyotrophic lateral sclerosis [Blauw et al., 2012]. While replication slippage and associated mechanisms [Chen et al., 2005; Pearson et al., 2005; McMurray, 2010] have been shown to cause the unstable contractions and expansions associated with classic trinucleotide repeat diseases, polyalanine expansions are hypothesized to be caused by unequal crossing-over [Warren, 1997; Brown and Brown, 2004]; however, this has not been proven in HOXA13 polyalanine expansion mutations in patients with HFGS. Replication slippage or slipped mispairing [Strand et al., 1993; Mortlock et al., 1996; Tishkoff et al., 1997; Innis et al., 2004; Chen et al., 2005; Parodi et al., 2008; Trochet et al., 2007] mediated by misalignment of short repetitive sequences during replication is another mechanism capable of generating polyalanine expansion (or contraction) mutations. Unequal sister chromatid exchange or homologous recombination is not supported by the observations of (1) polyalanine tract expansions involving at least two duplications in single alleles of HOXD13 and FOXL2 [Brown and Brown, 2004], (2) sequence incompatibilities with recombination in $P H O X 2 B$ [Trochet et al., 2007], and (3) lack of deletion products expected for unequal sister chromatid exchange in mosaic patients with polyalanine expansions in $P H O X 2 B$ [Trochet et al., 2007; Parodi et al., 2008]. We identified two rare cases of apparently de novo HFGS associated with HOXA13 polyalanine expansions allowing for experimental testing of these hypotheses.

\section{MATERIAL AND METHODS}

This study was approved by the University of Michigan Medical Institutional Review Board. Genomic DNA was extracted from leukocytes.

HOXA13 exons 1 and 2 were amplified by PCR using custom oligonucleotide primer pairs designed using Primer3 as previously described [Innis et al., 2004]. To obtain independent clones, PCR products were ligated into the p-GEM ${ }^{\circledR}$ T-Easy Vector system (Promega Corp., Fitchburg, WI), electroporated MegaX DH10 $\beta$ T1R Electrocomp ${ }^{\mathrm{TM}}$ cells (Invitrogen/Life Technologies Corp., Grand Island, NY), and blue-white colony selection was used.

We used SNPs upstream and downstream of HOXA13 and inverse PCR to determine SNP phase on each chromosome. BstBI restriction enzyme sites located outside of the region of interest were utilized to cut maternal genomic DNA and re-ligate, followed by inverse PCR to appose upstream and downstream SNPs. The PCR products were gel-excised and cloned. Sequencing of 18 clones using custom primers produced the final haplotypes (Fig. 3).

Luciferase assays were performed in NG108-15 cells comparing HOXA13 wt and HOXA13-G177R mutant proteins. The HOXA13G177R mutant construct was generated by using the QuikChange site-directed mutagenesis kit (Stratagene, La Jolla, CA), and the presence of this mutation was confirmed by DNA sequencing. NG108-15 cells (ATCC\#HB-12317) were maintained in DMEM media (Gibco/Life Technologies Corp., Grand Valley, NY) supplemented with 10\% FBS (Atlanta Biologicals, Norcross, GA), HAT (Invitrogen), and $1 \%$ penicillin/streptomycin. Cells $\left(1 \times 10^{5}\right)$ were seeded in 12 -well plates and grown for $24 \mathrm{hr}$ at $37^{\circ} \mathrm{C}$ with $5 \% \mathrm{CO}_{2}$. Transfections were performed using FuGENE6 transfec- tion reagent (Roche Applied Science, Indianapolis, IN), $0.1 \mu \mathrm{g}$ pRL-CMV Renilla, and $0.25 \mu \mathrm{g}$ pCAGGS-HOXA13 wild type or mutant, along with $0.5 \mu \mathrm{g}$ of a pGL4.23 plasmid (Promega) encoding luciferase and containing an EphA7 cis-regulatory element previously shown to be regulated by HOXA13 [Stadler et al., 2001]. Empty pGL4.23 and pCAGGs expression vectors were used as controls (red bars). The Dual-Glo Luciferase Assay system (Promega) was used to detect luciferase activity $24 \mathrm{hr}$ posttransfection in OptiPlate-96F black plates using a Fusion Microplate Analyzer (Perkin Elmer, Waltham, MA). Six replicates of each transfection were performed and the transfection assay was repeated a total of three separate times. Results were normalized for transfection efficiency using a Renilla luciferase expression vector as described by the manufacturer (Promega). Because the pGL4.23 vector also contains several HOXA13 binding sites, background activation of the empty pGL4.23 by HOXA13 was also subtracted from the final luciferase levels after Renilla normalization.

\section{CLINICAL REPORTS}

Patient 1 is a female born to nonconsanguineous parents. No pregnancy or birth history was available. The patient had a history of normal developmental milestones. We first evaluated her at 17 years, 10 months of age. At that time, her height was 150.2 centimeters ( $\sim 3 \mathrm{rd}$ centile), her weight was $45.7 \mathrm{~kg}$ (5-10th centile), and her head circumference was $54.0 \mathrm{~cm}$ ( $\sim 35$ th centile). The patient's medical history was significant for genitourinary abnormalities including a small bladder, urinary leakage, and multiple urinary tract infections necessitating numerous Mitrofanoff and bladder augmentation procedures. Additional medical history was significant for back and heel pain, a right inguinal hernia statuspostrepair, a left heminephrectomy, and scoliosis with no thoracolumbar vertebral segmentation anomaly. Clinical examination and radiographic findings showed a short distal phalanx of the thumbs bilaterally, micronychia of the thumbs bilaterally, limited flexion of the DIP joint and distal phalanges of the fourth fingers bilaterally, radial fifth finger clinodactyly bilaterally, short distal phalanges of all toes bilaterally, short middle phalanges of all toes bilaterally, fusion of the middle and distal phalanges of all toes bilaterally, hypoplasia of the cuneiform bilaterally with right-sided fusion with the metatarsal, and micronychia of the great toes bilaterally (Fig. 1A). Based on these historical and physical findings, the patient was diagnosed with HFGS. The patient's mother and maternal half-sister were also examined and were unaffected (Fig. 1B). The patient's father is deceased but reportedly did not have thumb, toe, or genitourinary abnormalities. A maternal halfbrother and paternal half-brother were also both reportedly unaffected. Based on the family history and physical exams, we hypothesized that the first patient had de novo HFGS.

Patient 2 is a male born to a 25-year-old mother and 27-year-old father who are nonconsanguineous. The patient was born at 39 weeks' gestation and weighed $4.1 \mathrm{~kg}$ (>90th centile) and was $51.4 \mathrm{~cm}$ long (75-90th centile). The pregnancy was uncomplicated. Shortly after birth, the patient was diagnosed with bacterial pneumonia and spent time in the neonatal intensive care unit. The patient met expected developmental milestones. We first evaluated him at 35 months of age. At that time, his height was 94.8 


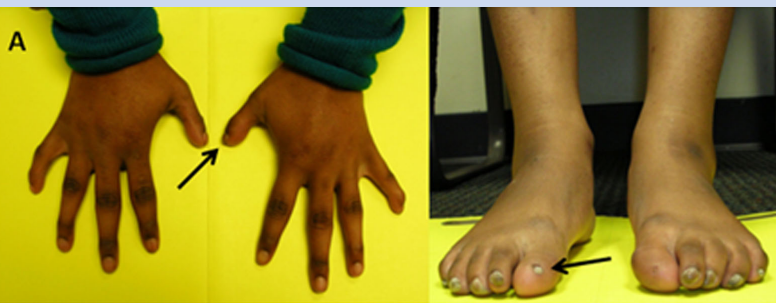

B

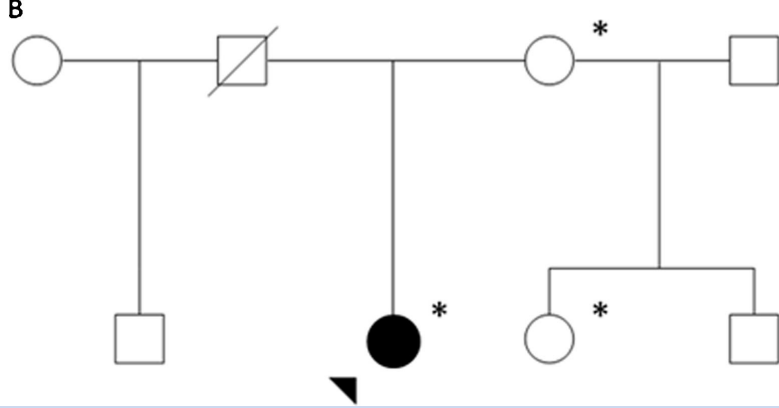

FIG. 1. Phenotype and pedigree of Patient 1. A: Limb phenotype in Patient 1 with hand-foot-genital syndrome (HFGS). Note the short thumbs and great toes with micronychia of the thumb and great toe bilaterally [arrows]. B: Pedigree of the patient's family. Open squares or circles mean the individual is unaffected. An asterisk indicates individuals who were clinically examined.

centimeters (46th centile), his weight was $13.8 \mathrm{~kg}$ (40th centile), and his head circumference was $49.8 \mathrm{~cm}$ (54th centile). Physical examination revealed several bony abnormalities of the hands and feet, as well as partial cutaneous two-three-toe syndactyly bilaterally (Fig. 5C,D). The patient was also found to have mild glandular hypospadias with no history of urinary tract infections and occasional toe-walking. A skeletal survey showed the following abnormalities: short middle phalanx of all fingers, short proximal and distal phalanges of the thumbs, radial fifth finger clinodactyly bilaterally, short first metacarpals bilaterally, short distal phalanges of all toes bilaterally, short proximal phalanges of the great toes, absent ossification of the middle phalanges of toes 2 through 5 bilaterally, and short first metatarsals. Based on these findings, the patient was diagnosed with HFGS.

The patient's mother, father, brother, sister, maternal grandfather, and maternal grandmother were also examined and were unaffected. Based on the family history and physical exams, we hypothesized that the second patient also had de novo HFGS.

\section{RESULTS}

\section{Patient 1}

Amplification of an exon 1 segment including the large 18 residue polyalanine tract (III) resulted in one product compatible with wild type length (arrow, Fig. 2A) and a larger amplicon. Sequencing of independent clones from several independent amplifications showed one allele with a nine-alanine expansion in tract III (c.357_383 dup27, Fig. 2B) and, surprisingly, a second allele with a point mutation, c.529G $>$ C (p.Gly177Arg) (Genbank accession no. JN628255; Fig. 2C), showing the two sequence variations on separate homologs.

To determine if either mutation was inherited, maternal genomic DNA from two independent blood samples was analyzed for the presence of the nine-alanine expansion and the c.529G $>C$ point mutation identified in Patient 1, which showed that the mother carried neither. While the mother could still be a low-level somatic or germline mosaic, neither mutated allele, nor any shorter HOXA13 PCR products, were detected in repeated amplifications of leukocyte derived DNA. A SNP-based haplotype upstream and downstream of the HOXA13 gene was constructed for Patient 1 and her mother to determine the parental origin of the polyalanine expansion. Patient 1's HOXA13 SNP haplotype showed that the polyalanine expansion must have originated de novo on the maternal chromosome while the point mutation must have originated on the paternally inherited chromosome (Fig. 3).

To determine whether the polyalanine expansion occurred as a result of unequal crossing-over between homologs during meiosis, we used inverse PCR. Patient 1 was homozygous for the upstream SNPs rs7812039 and rs13243033, whereas her mother was heterozygous at these loci. Figure 3 also shows the results of inverse PCR with maternal DNA. The de novo polyalanine expansion did not arise by unequal crossing-over between maternal chromosome 7 homologs, and must have occurred as a result of either slipped mispairing or unequal sister chromatid exchange in maternal mitosis prior to gamete production or during meiosis II, or by either mechanism early on postzygotically. Although we did not examine for mosaicism in Patient 1, a deleted product by PCR analysis was never detected, suggesting that if the expansion had occurred postzygotically, unequal sister chromatid exchange was unlikely.

Given that homozygous loss of Hoxa13 function is lethal [Mortlock et al., 1996; Warot et al., 1997; Shaut et al., 2008], and HOXA13 polyalanine tract expansions lead to a loss of function [Innis et al., 2004], it is unlikely that Patient 1 would be compound heterozygous for two disease-causing mutations. However, the c.529G > C (p.Gly177Arg) point mutation found in Patient 1 has not been previously reported in dbSNP (build 135) or in the NHLBI Exome Sequencing Project (snp.gs.washington.edu/EVS/) and is therefore a very rare variant. Additionally, the glycine residue at position 177 is highly conserved in HOXA13 among vertebrates [Mortlock et al., 2000; Lehoczky and Innis, 2008] and lies within a MEIS1 interacting domain [Williams et al., 2005]. Finally, arginine (basic, hydrophilic) is not a conservative substitution for glycine (small, neutral). Polyphen-2 analysis (genetics.bwh.harvard.edu/ pph2/) showed this substitution to be "probably damaging" with a score of 0.992 . Analysis of the c.529G $>$ C point mutation using Genomic Evolutionary Rate Profiling (GERP) [Cooper et al., 2005] showed a rejected substitution score of 3.56, suggesting evolutionary selection against substitution at this nucleotide.

Therefore, to test the effect of this sequence variation on function, the p.Gly177Arg mutation was introduced into a plasmid containing the mouse Hoxa13 cDNA through site-directed mutagenesis and then the p.Gly177Arg HOXA13 transcriptional activation function was measured relative to WT HOXA13 on the EphA7 promoter in vitro. The EphA7 promoter is regulated in vivo by Hoxa13 [Stadler et al., 2001]. As seen in Figure 4, the p.Gly177Arg 


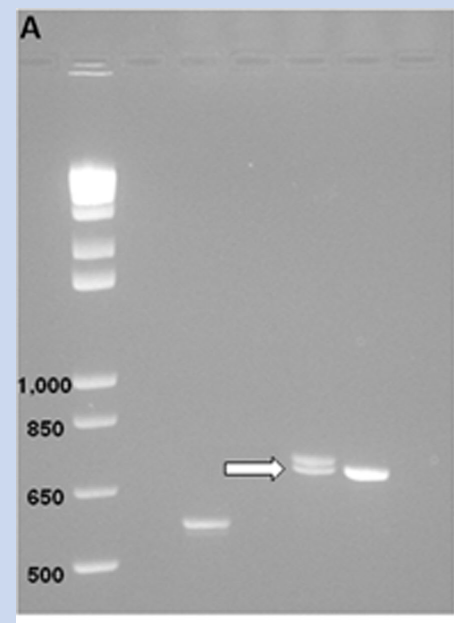

bp

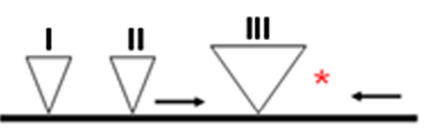

I, II, III-polyalanine repeats *G177R
B Polyalanine repeat III

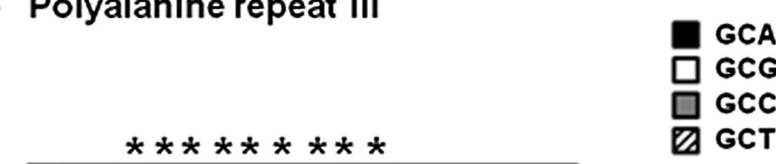

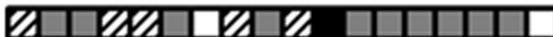

Non-expansionallele

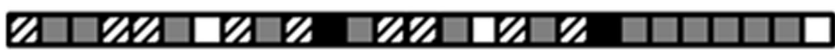

Expansionallele

C G177R

"Expansionallele" (WT seq)

GGCAGC GGC TAC TAC
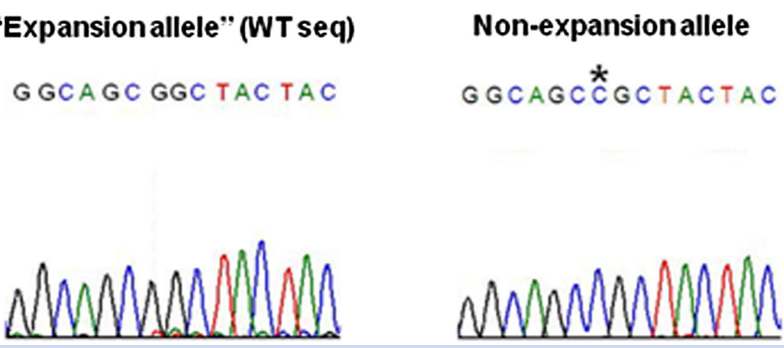

FIG. 2. Polyalanine expansion and rare variant in Patient 1. A: The PCR and clone sequencing analysis of HOXA13 polyalanine tract III. Lane 1: Patient 1 PCR showing two products, one corresponding with the WT allele [arrow] and the other with the expansion. Lane 2: WT DNA. The PCR product visualized adjacent to the marker lane was a separate control reaction. B: Sequencing of cloned expansion products in the patient showed a 27 bp (nine-alanine) tandem duplication (c.35?_383 dup27). Imperfect GCN triplets encoding alanine residues are depicted as shown; ${ }^{*}$ nine-alanine triplet codons duplicated in the expansion allele. C: Sequencing downstream of the polyalanine tract III showed a point mutation (c.529G $>$ C, p.Gly177Arg; Genbank accession no. JN628255) in the allele not carrying the polyalanine expansion. The WT sequence is shown on the left and the asterisk indicates the mutated base in a PCR clone from the nonexpansion allele [c.529G $>C$ ].

mutation did not significantly affect transcriptional activation by HOXA13 in this in vitro assay $(P=0.631)$. This result does not preclude an effect of this amino acid substitution on regulatory activity at other target loci in different cellular contexts, but it underscores the importance of functional analysis of sequence variants. Also, if Patient 1's father was heterozygous for the p.Gly177Arg variant, these results would be consistent with absence of paternal HFGS features.

\section{Patient 2}

The PCR analysis of large repeat tract III followed by cloning and sequencing showed Patient 2 to carry one 6-alanine expansion allele (c.358_375 dup18, inherited from his father) and a 10-alanine contraction allele (c.355_384 del30, inherited from his asymptomatic mother and her asymptomatic father). Close examination of Patient 2's father showed a normal extremity exam (Fig. 5A,B), suggesting either lack of penetrance or mosaicism. Paternal and maternal HOXA13 PCR sequences matched Patient 2's two alleles (Fig. 5E,F). A SNP-based haplotype analysis upstream and downstream of HOXA13 was performed to identify the paternal chromosome carrying the expanded alanine tract (Fig. 6). Both unaffected siblings carried the same paternal allele as Patient 2 without the expanded alanine tract, suggesting that the father was mosaic for the expansion. The PCR sequencing on the paternal grandparents showed that neither were carriers, supporting the hypothesis that Patient 2's father sustained a postzygotic polyalanine expansion.

To prove mosaicism and to distinguish unequal sister chromatid exchange from slipped mispairing, a skin biopsy was obtained from the father to look for WT, expanded, and contracted polyalanine tract III alleles. One week later, after minimal expansion, the fibroblasts were harvested, and a sample was plated into ten 96well plates at an average concentration of 0.8 cells per well. Many wells showed no growth, but genomic DNA from 58 wells was harvested and subjected to HOXA13 PCR. The DNA from 11 clones failed to amplify. Thirty-four clones had both an expansion allele and a normal allele; 10 clones showed only amplification of the normal sized allele. Thus, 34/44 clones (77\%) showed expansion and normal alleles, and 10/44 clones (23\%) had normal alleles only, by PCR. A separate cloning experiment performed later after further expansion of the original culture, plated at higher density, detected no contracted allele among 159 samples. An additional paternal DNA sample derived from saliva showed only six-alanine expansion allele and normal allele products by PCR. No shorter sixalanine contraction allele product was observed in any genomic PCR with paternal DNA from any tissue or with any of the clonal fibroblast isolates. 


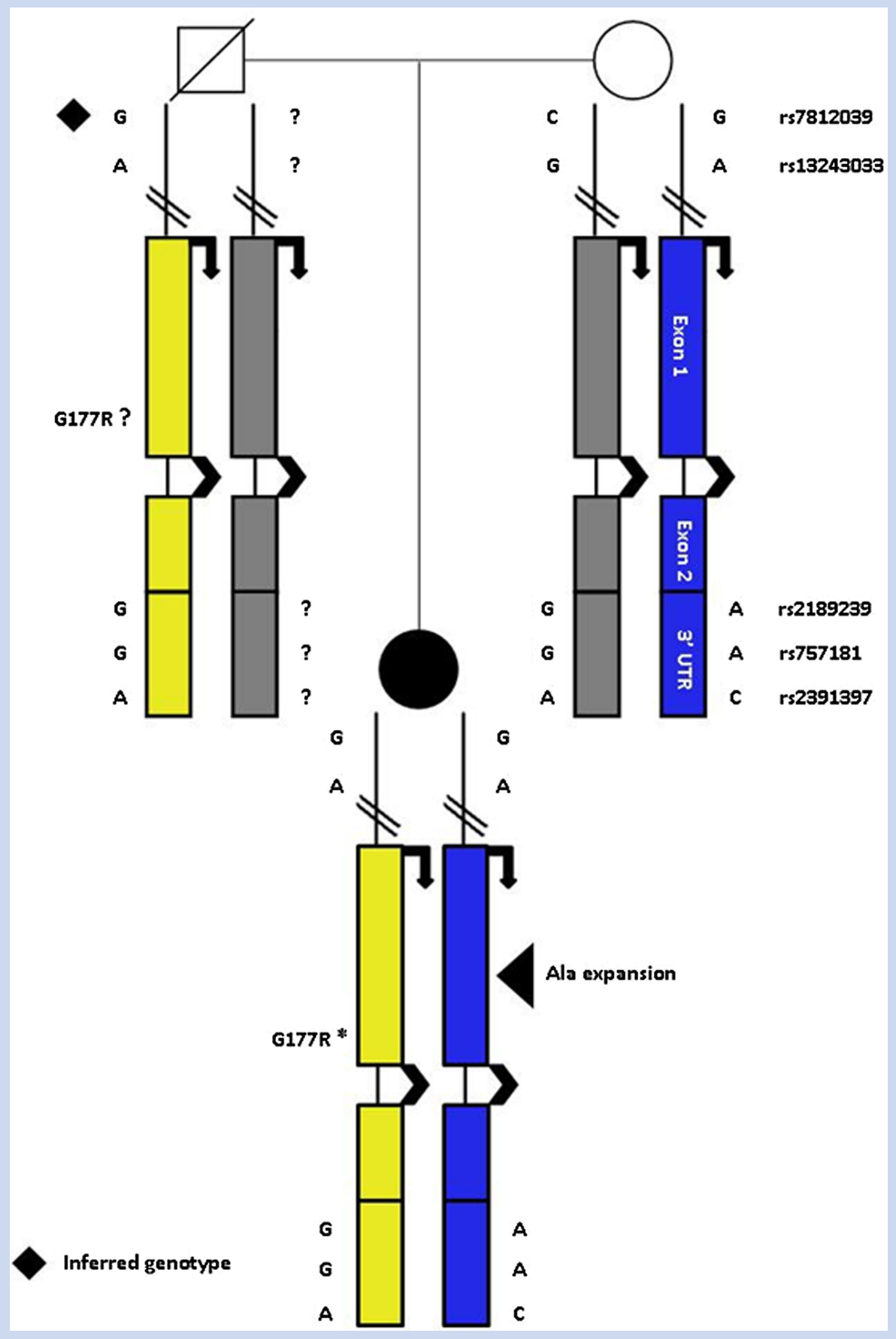

FIG. 3. Haplotypes of Family 1. Haplotype analysis of Patient 1 showed that the polyalanine expansion occurred on the maternally inherited allele, while the p.Gly177Arg mutation originated on the paternally inherited allele. Diamond refers to inferred haplotypes. SNPs and hg19 reference genomic positions are: rs7812039 (chr7:27,248,891], $\sim 9.2 \mathrm{~kb}$ centromeric (upstream] of the $5^{\prime}$ end of H0XA13; rs 13243033 [chr7:27248373), $\sim 8.7$ kb centromeric of HOXA13; rs2189239 (chr7:27237453), $\sim 2.2 \mathrm{~kb}$ telomeric of the HOXA13 $5^{\prime}$ end; rs757181 (chr7:27236958), 2.7 kb telomeric of the HOXA13 $5^{\prime}$ end; and rs239139? (chr7:27236558), $\sim 3.1 \mathrm{~kb}$ telomeric of the HOXA13 $5^{\prime}$ end. 


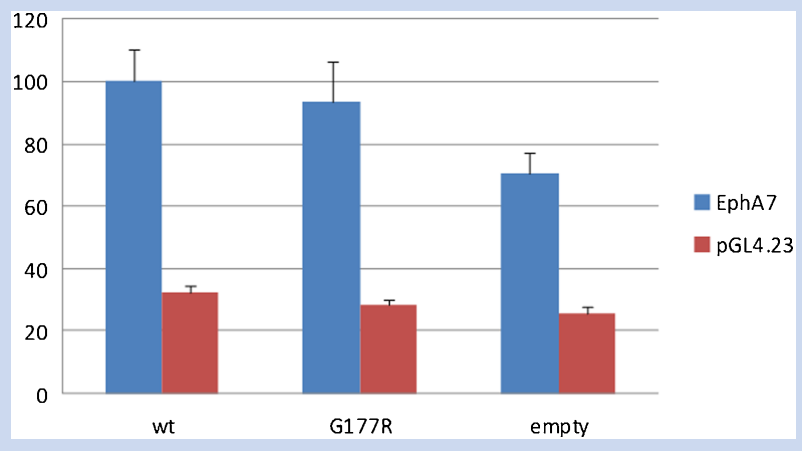

FIG. 4. In vitro transcriptional activation function of p.Gly177Arg HOXA13 on the EphA7 promoter. Percent luciferase activities [relative to wt control] are indicated on the $y$-axis. HOXA13 wt or G177R (normalized to Renilla and empty vector controls) are plotted on the $x$-axis. Results are the mean \pm SD of three separate experiments.

\section{DISCUSSION}

Family 2 is the most informative regarding mechanism(s) for HOXA13 polyalanine expansions. From four different sites (blood, skin, saliva, and gonads by inheritance) and from individual fibroblast clones of skin, only the expanded and normal allele sizes were observed, supporting somatic and gonadal mosaicism in the asymptomatic father of Patient 2. In addition, if the mechanism of the expansion was unequal crossing over between sister chromatids, there should have been three different paternal cell lines: a sixalanine expanded/WT, WT/WT and a six-alanine contracted/WT. That no six-alanine contracted product was observed indicates that either a cell line with a six-alanine contraction/WT genotype was selected against in vivo or in vitro or that the mutational mechanism is not unequal sister chromatid exchange. The former explanation seems very unlikely given that a 10 -alanine contraction allele is a low frequency polymorphism in the normal population, and indeed survives quite well in Patient 2, his mother, and maternal grandfather, making it difficult to argue that heterozygous loss of 6-alanine residues would be deleterious. Thus, slipped mispairing appears most likely as the mechanism of the postzygotic expansion in the mosaic father. Unequal crossing-over between homologs [Warren, 1997; Brown and Brown, 2004], which is also theoretically possible as a mechanism of polyalanine expansion, was not supported by the data from either of the patients described here.

Arai et al. [2007] proposed that unequal sister chromatid exchange may have led to de novo polyalanine expansions in the PHOX2B gene in patients with congenital central hypoventilation syndrome (CCHS; OMIM 209880); however, no data proving this mechanism was presented. The authors proposed that this mechanism could account for the stability of the polyalanine expansions as well as the presence of normal contraction alleles. Contraction alleles, although quite rare, have also been observed as presumably normal variants in the HOXA13 gene [Brown and Brown, 2004;
Innis et al., 2004; Innis, 2006] and other genes with polyalanine repeats. In Family 1, we observed no mosaicism for the polyalanine expansion (and the expected reciprocal deleted product) in either maternal or proband DNA. Failure to observe the deleted product does not eliminate the possibility of either unequal sister chromatid exchange or slipped mispairing occurring in the maternal germline.

However, our data in Family 2 strongly support slipped mispairing during replication as the mechanism for this instance of HOXA13 polyalanine expansion. Slipped mispairing was previously proposed as a mechanism for the instability of repetitive DNA tracts in yeast mutants for mismatch repair genes [Strand et al., 1993]. Trochet et al. [2007] also presented a family where unequal sister chromatid exchange was not a suitable explanation for a PHOX2B polyalanine expansion in a mosaic (leukocyte DNA) parent due to failure to detect a deleted product, and they proposed that a mispairing event without repair was more likely. Parodi et al. [2008] also documented somatic mosaicism for PHOX2B expansions, and did not identify shorter products expected for unequal sister chromatid exchange in mosaic individuals. Additional polyalanine tract expansions involving at least two duplications in single alleles of HOXD13 and FOXL2 [Brown and Brown, 2004], also support slipped mispairing. By replication slippage, both expansions and contractions could occur, depending on whether template or nascent strand misalignment was involved [Strand et al., 1993; Chen et al., 2005; McMurray, 2010]. Finally, Cocquempot et al. [2009] suggested FosTES (Fork stalling and TEmplate Switching) [Lee et al., 2007] as the mechanism for polyalanine expansion in the Hoxd13 gene in the mouse mutant $D y c$, whose structure is quite different from typical HOXA13 or HOXD13 polyalanine expansions. The authors stated that polyalanine tracts contain microhomology, symmetry, and specific sequence motifs that commonly lead to FosTES.

In our report, we have proven that slipped mispairing during replication must have occurred in Family 2 and is possible in Family 1. Whereas paternal mosaicism in Family 2 indicates a postzygotic origin for the expansion, the timing of expansion in Family 1 is unclear. In addition, the 10-alanine contraction in Proband 2 was inherited from the maternal grandfather. Thus, not unlike polyalanine expansions, this contraction has been stable in this family through at least three generations. However, it would be intriguing to examine for mosaicism of contractions as well, since an expanded allele would be an expected product of unequal sister chromatid exchange.

We reported on the molecular basis of Hypodactyly in mice, a 50 base pair deletion in Hoxa13, and proposed that the deletion arose from slipped mispairing or unequal crossing over catalyzed by nearby 10 base pair GC-rich microhomology in the first exon of Hoxa13 [Mortlock et al., 1996]. The location of human polyalanine expansions as well as the Hypodactyly deletion in HOXA13 exon 1 associated with numerous GC-rich microhomologies supports the hypothesis that the structure predisposes to expansions, contractions, and deletions of several types depending on the nature of the mispairing event.

Finally, other than for PHOX2B [Parodi et al., 2008; Trochet et al., 2007], this is the third investigation of mosaic polyalanine expansion carriers documenting results consistent with slipped 

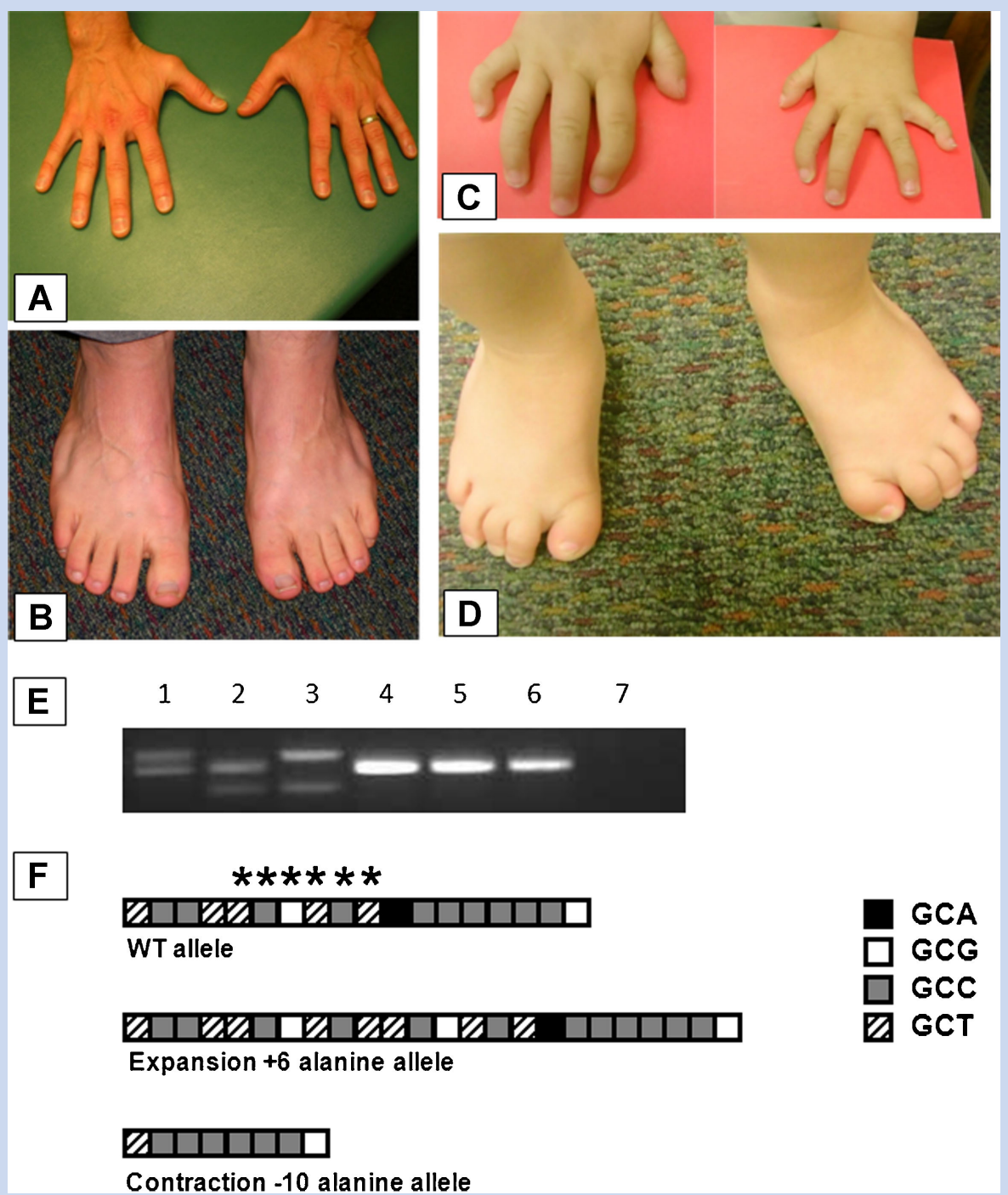

FIG. 5. Phenotype and polyalanine repeat size in Family 2. A,B: Hands and feet of asymptomatic father of Patient 2. C,D: Hands and feet of Patient 2 demonstrating typical HFGS skeletal features. E: HOXA13 PCR products across large repeat III in Family 2. Lane 1, asymptomatic father [6-alanine expansion (c.358_375 dup18)/WT alleles]; lane 2, asymptomatic mother [10-alanine contraction (c.355_384 del30)/WT); lane 3, Patient 2 (6alanine expansion/10-alanine contraction); lanes 4,5, asymptomatic sister and brother (WT/WT); lane 6, normal control; lane ?, water control. F: Imperfect GCX triplets encoding alanine residues are depicted as shown; * six-alanine triplet codons duplicated in the expansion allele. The contraction allele sequence of triplet codons inherited from the maternal grandfather is shown.

mispairing. In contrast, despite the possibility of unequal sister chromatid exchange or homologous recombination generating polyalanine expansions, there has been no report of a proven occurrence. It will be important to identify mosaic individuals for other polyalanine expansion disorders, and possibly for contractions, and investigate particularly by clonal analysis whether replication slippage is the predominant mechanism or not.

\section{WEBSITE RESOURCES}

OMIM: http://www.ncbi.nlm.nih.gov/
GERP calculation: http://gvs.gs.washington.edu/SeattleSeq Annotation/

Polyphen-2: http://genetics.bwh.harvard.edu/pph2/

NHLBI Exome Sequencing Project: http://snp.gs.washington. edu/EVS/

UCSC Genome Browser: http://genome.ucsc.edu/dbSNP135: http://www.ncbi.nlm.nih.gov/snp/

SIFT: http://sift.jcvi.org/

Primer3: http://frodo.wi.mit.edu/primer3/

GeneReviews: http://www.ncbi.nlm.nih.gov/sites/GeneTests/ review? $\mathrm{db}=$ GeneTests 


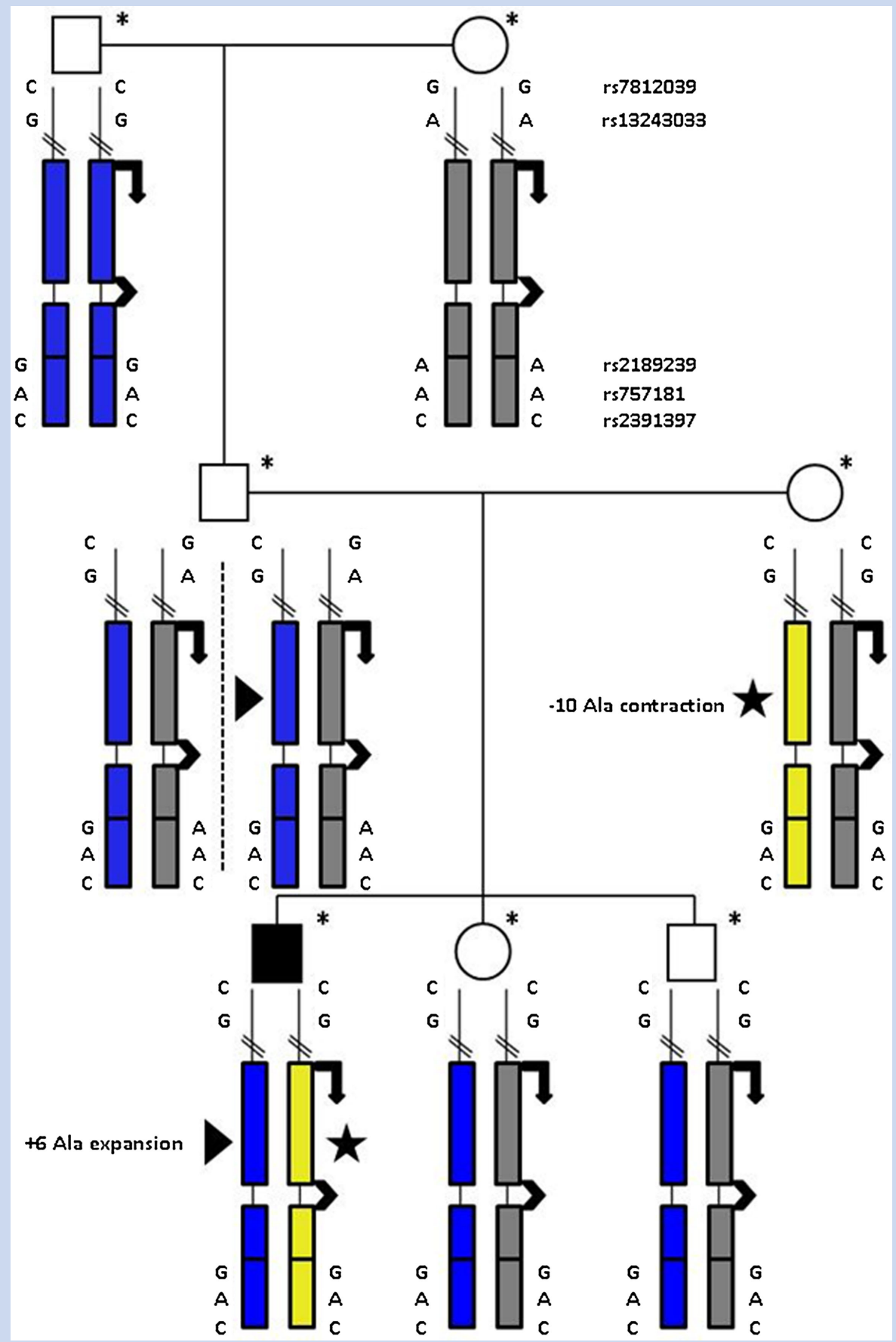

FIG. 6. Pedigree and haplotypes of Family 2. Patient 2 with HFGS, filled in square. Dotted line: father of proband is mosaic for HOXA13 polyalanine expansion/WT and WT/WT allele-bearing cells. Patient 2 inherited a 10-alanine contraction (instead of 18 alanines, this allele has 8 -alanine residues in tract III] from his asymptomatic mother. All individuals with an * were examined. Asymptomatic siblings of Patient 2 inherited the same chromosome haplotype from their father, without the polyalanine expansion observed in Patient 2.

\section{ACKNOWLEDGMENTS}

The authors thank the patients and their families for their research participation. We thank Dr. Tom Glover for helpful comments.
This research was funded by the University of Michigan, Department of Human Genetics and the Department of Pediatrics. Dr. Innis is the Morton S. and Henrietta K. Sellner Professor of Human Genetics. 


\section{REFERENCES}

Arai H, Otagiri T, Sasaki A, Hashimoto T, Umetsu K, Tokunaga K, Hayasaka K. 2007. De novo polyalanine expansion of PHOX2B in congenital central hypoventilation syndrome: Unequal sister chromatid exchange during paternal gametogenesis. J Hum Genet 52:921-925.

Blauw HM, van Rheenen W, Koppers M, Van Damme P, Waibel S, Lemmens R, van Vught PWJ, Meyer T, Schulte C, Gasser T, Cuppen E, Pasterkamp RJ, Robberecht W, Ludolph AC, Veldink JH, van den Berg LH. 2012. NIPA1 polyalanine repeat expansions are associated with amyotrophic lateral sclerosis. Hum Mol Genet 21:2497-2502.

Brown LY, Brown SA. 2004. Alanine tracts: The expanding story of human illness and trinucleotide repeats. Trend Genet 20:51-58.

Chen J-M, Chuzhanova N, Stenson PD, Ferec C, Cooper DN. 2005. Metaanalysis of gross insertions causing human genetic disease: Novel mutational mechanisms and the role of replication slippage. Hum Mutat 25:207-221.

Cocquempot O, Brault V, Babinet C, Herault Y. 2009. Fork stalling and template switching as a mechanism for polyalanine tract expansion affecting the DYC mutant of Hoxd13, a new murine model of synpolydactyly. Genetics 183:23-30.

Cooper GM, Stone EA, Asimenos G, Comparative NISC Program Sequencing, Green ED, Batzoglou S, Sidow A, 2005. Distribution and intensity of constraint in mammalian genomic sequence. Genome Res 15:901-913.

Goodman FR, Fryns J-P, Mortlock DP, Innis JW, Holmes LB, Donnenfeld AE, Feingold M, Beemer FA, Hennekam RCM, Scambler PJ. 2000. Novel HOXA13 mutations and the phenotypic spectrum of hand-foot-genital syndrome. Am J Hum Gen 67:197-202.

Innis JW. Hand-foot-genital syndrome. In: Pagon RA, Bird TC, Dolan CR, Stephens K, editors. GeneReviews [Internet]. Seattle, WA: University of Washington Seattle; 2006.

Innis JW, Mortlock D, Chen Z, Ludwig M, Williams ME, Williams TM, Doyle CD, Shao Z, Glynn M, Mikulic D, Lehmann K, Mundlos S, Utsch B. 2004. Polyalanine expansion in HOX A13: Three new affected families and the molecular consequences in a mouse model. Hum Mol Genet 1322:2841-2851.

Lee JA, Carvalho CMB, Lupski JR. 2007. A DNA replication mechanism for generating nonrecurrent rearrangements associated with genomic disorders. Cell 131:1235-1247.

Lehoczky J, Innis JW. 2008. Expanded HOXA13 polyalanine tracts in a monotreme. Evol Dev 10:433-438.
McMurray CT. 2010. Mechanisms of trinucleotide repeat instability during human development. Nat Rev Genet 11:786-799.

Mortlock DP, Innis JW. 1997. Mutation of HOXA13 in hand-foot-genital syndrome. Nat Genet 15:179-180.

Mortlock DP, Post LC, Innis JW. 1996. The molecular basis of Hypodactyly $(H d)$ : A deletion in Hoxa13 leads to arrest of digital arch formation. Nat Genet 13:284-289.

Mortlock DP, Sateesh P, Innis JW. 2000. Evolution of N-terminal sequences of the vertebrate HOXA13 protein. Mamm Genome 11:151-158.

Parodi S, Bachetti T, Lantieri F, Di Duca M, Santamaria G, Ottonello G, Matera I, Ravazzolo R, Ceccherini I. 2008. Parental origin and somatic mosaicism of PHOX2B mutations in congenital central hypoventilation syndrome. Hum Mutat 29:206-215.

Pearson CE, Edamura KN, Cleary JD. 2005. Repeat instability: Mechanisms of dynamic mutations. Nat Rev Genet 6:729-742.

Shaut CAE, Keene DR, Sorensen LK, Li DY, Stadler HS. 2008. HOXA13 is essential for placental vascular patterning and labyrinth endothelial specification. PLoS Genet 4:e1000073.

Stadler HS, Higgins KM, Capecchi MR. 2001. Loss of Eph-receptor expression correlates with loss of cell adhesion and chondrogenic capacity in Hoxa13 mutant limbs. Development 128:4177-4188.

Strand M, Prolia TA, Liskay RM, Petes TD. 1993. Destabilization of tracts of simple repetitive DNA in yeast by mutations affecting DNA mismatch repair. Nature 365:274-276.

Tishkoff DX, Filosi N, Gaida GM, Kolodner RD. 1997. A novel mutation avoidance mechanism dependent on S. cerevisiae RAD27 is distinct from DNA mismatch repair. Cell 88:253-263.

Trochet D, de Pontual L, Keren B, Munnich A, Vekemans M, Stanislas L, Amiel J. 2007. Polyalanine expansions might not result from unequal crossing-over. Hum Mutat 28:1043-1044.

Warot X, Fromental-Ramain C, Fraulob V, Chambon P, Dollé P. 1997. Gene dosage-dependent effects of the Hoxa-13 and Hoxd-13 mutations on morphogenesis of the terminal parts of the digestive and urogenital tracts. Development 124:4781-4791.

Warren ST. 1997. Polyalanine expansion in synpolydactyly might result from unequal crossing-over of HOXD13. Science 275:408409.

Williams TM, Williams ME, Innis JW. 2005. Range of HOX/TALE superclass associations and protein domain requirements for HOXA13-MEIS interaction. Dev Biol 277:457-471. 